\title{
Comparison of Passive and Active Methods for Minimization of Sound Radiation by Vibrating Clamped Plate
}

\author{
M.M. KOZUPA* AND J.W. WiCIAK \\ Department of Mechanics and Vibroacoustics, AGH University of Science and Technology \\ al. A. Mickiewicza 30, 30-059 Kraków, Poland
}

\begin{abstract}
The paper is an analytical and experimental study of a smart structure consisting of steel plate with bonded piezoelectric transducers and porous elastomer layer. Active control of sound radiation from a plate clamped at the edge square is examined. Simulations and numerical computation of the experiment are performed in Ansys environment. Calculations of plate vibration and sound radiation under stepped harmonic force are performed. The experimental setup consists of two rooms with the test opening in between. A variety of test cases were studied for three different configurations: steel plate + piezoelectric transducer, steel plate + elastomer layer, steel plate + piezoelectric transducer + elastomer layer. The aim of the paper is to illustrate the possibilities of using piezoelectric materials as an active control with elastomer layers as passive methods in one structure to improve the transmission loss.
\end{abstract}

PACS: 43.40.Dx, 43.40.Rj, 43.40.Vn, 43.50.Gf, 43.50.Ki

\section{Introduction}

Transmission loss is a function of various different parameters e.g. thickness, density, Young's modulus, Poisson's ratio, void ratio and many more. There is also a frequency dependence on transmission loss: resonant frequencies (low band), mass law (medium band), coincidence frequency (high band). For sound insulation the mas law states, that insulation states that doubling mass we increase sound insulation $6 \mathrm{~dB}$ and is expressed by the formula

$$
R=10 \log \left|1+\mathrm{j} \frac{\omega m}{2 \rho c}\right|^{2} \cong 20 \log \left(\frac{\pi f m}{\rho c}\right),
$$

where $m$ is unit weight of the panel or wall. It can be a good rough approach to calculate sound insulation up to coincidence frequency. These conventional technique of additional mass tends to be ineffective at low frequencies and can be difficult to predict their precise effect. Defining the density of the material a void ratio can be added in terms of porous materials.

The air molecules in the interstices of the porous material oscillate with the frequency of the exciting sound wave, this oscillation results in frictional losses. Changes in flow direction, expansions and contractions of the flow throughout irregular pores result in a loss of momentum in the direction of wave propagation. These two phenomena account for most of the energy losses in the high-frequency range $[1,2]$. However, a porous layer can

\footnotetext{
* corresponding author; e-mail: Michal.Kozupa@agh.edu.pl
}

absorb a large part of acoustic energy only if its thickness is comparable to the wavelength of the incident sound. Considering, an incident sound of about $200 \mathrm{~Hz}$ when the wavelength of sound in air is about $1.715 \mathrm{~m}$ a porous material needs to be $0.43 \mathrm{~m}$ thick. This is due to the theory that it should be of a thickness about a quarter of the wavelength of the incident sound. This implies that such a passive technique is not very efficient in the low-frequency region. In general there are two types of active-passive noise control [3]. The adaptive passive techniques use devices whose static properties are changed. The hybrid techniques use active and passive elements in parallel. Active components are used to enhance the performance of the passive device which usually carries the primary vibration attenuation characteristic $[4,5]$. In this paper not only additional mass is considered to increase sound insulation but also piezoelectric transducer (PZT) as active methods.

\section{Experimental setup}

Two different test plates are used to examine in three configurations. First is steel plate with bonded PZTs, second is steel plate with bonded PZTs but with additional porous $2.5 \mathrm{~mm}$ thick elastomer layer. A variety of test cases were studied for three different configurations: steel plate + PZT, steel plate + elastomer layer, steel plate + PZT + elastomer layer. Both plates have nine piezoceramic patches placed on the plane. Eight PZTs are distributed symmetrically in the middle of each quarter: SET I (PZT no. 1, 2, 3, 4), SET II (PZT no. $5,6,7,8)$. The remaining PZT located near the centre 
is used as a mechanical force and excites the test plate to vibrate. Placement of PZT patches was considered under assumption of maximum displacement for the first radiation modes [6-12].

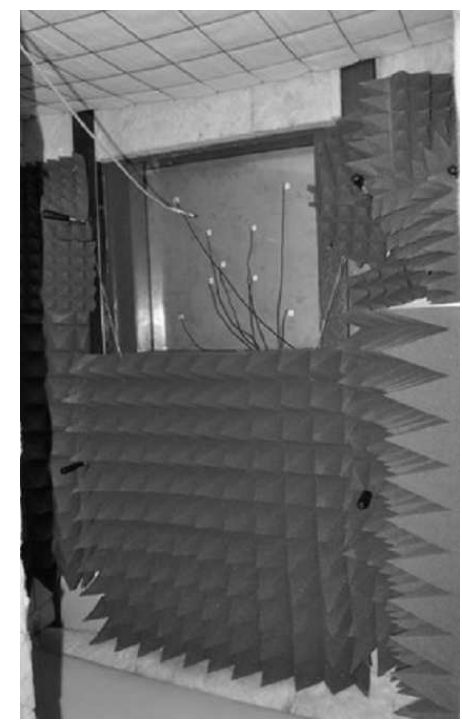

Fig. 1. Receiving chamber with the test specimen.

Existing chamber was modified and a sound-absorbing enclosure was built to obtain semi-anechoic conditions illustrated in Fig. 1. The test plate is mounted in the test opening so that the elastomer layer is on the sending chamber side of the experimental setup. The layer is porous with the void ratio of 0.5 and thickness of $2.5 \mathrm{~mm}$. Boundary conditions of the test plate are clamped, rigidly around the edges. The test specimen is placed in the opening between coupled reverberant chambers for sound insulation measurements.

The acoustical conditions produced by sound absorbing panels allow to measure near field sound radiation. Homogeneous acoustic field of white noise was created in the sending chamber to measure transmission loss. For active methods efficiency measurements single frequencies as pure tone sound wave are used to excite the test plate. Signal acquisition and control of PZT tiles is realized via multifunction data acquisition card and LabView environment.

\section{FE model}

To identify radiation modes and vibration causing structure borne sound, finite element (FE) model is built in Ansys software. Entering in FE-model the exact parameters of piezoceramics including permittivity, elastic compliance and piezoelectric charge constant voltage are used as an input parameter. Additionally, acoustic volume is modelled on both sides of the test structure. In this case pressure is the input parameter to simulate incident wave from sending chamber.

Figure 2 shows test plate and air volume behind plate which is the source of acoustic excitation. A volume is

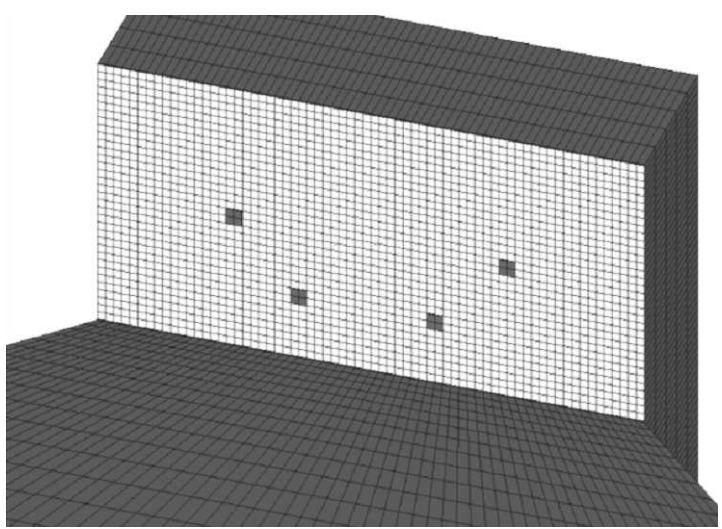

Fig. 2. FE model of plate and surrounding air.

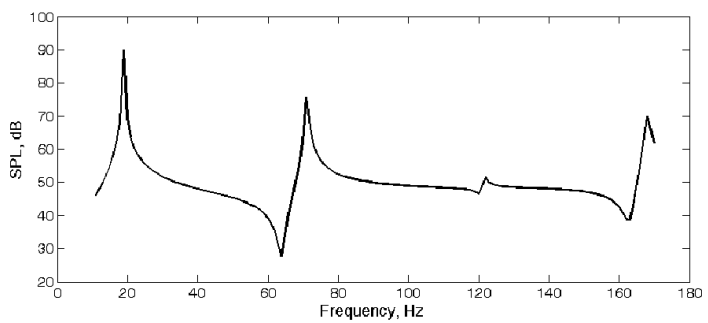

Fig. 3. Sound pressure level for test plate acoustic radiation.

modelled in front of test plate to simulate acoustic radiation. Sample graph of sound pressure level in node placed $0.5 \mathrm{~m}$ from the test plate is presented in Fig. 3 .

\section{Measurements and comparison}

\subsection{Sound insulation measurements}

The first measurements are performed to observe the passive technique effect of elastomer layer on transmission loss. Using white noise sound in the sending chamber sound transmitted to the receiving semi-anechoic chamber is measured. The resulting transmission loss levels in frequency domain are presented in Fig. 4. It can be seen that at frequencies below $100 \mathrm{~Hz}$ the influence of additional mass is negligible or even negative at resonant frequencies. Above $400 \mathrm{~Hz}$ transmission loss is increasing steadily but resulting only in $R=1 \mathrm{~dB}$ of sound reduction index in whole spectrum.

Sound insulation measurements confirm the theory explained in introduction. Small increase of mass of about $15 \%$ and thin porous layer slightly affected on transmission loss.

\subsection{Active structural acoustic control}

In the preliminary research, mechanical force input is used to excite the test plate to vibrate. The mechanical force is harmonic voltage signal applied on 9th PZT (Fig. 5) element located near the centre of the test plate resulting a bending moment. Eight other PZT patches 


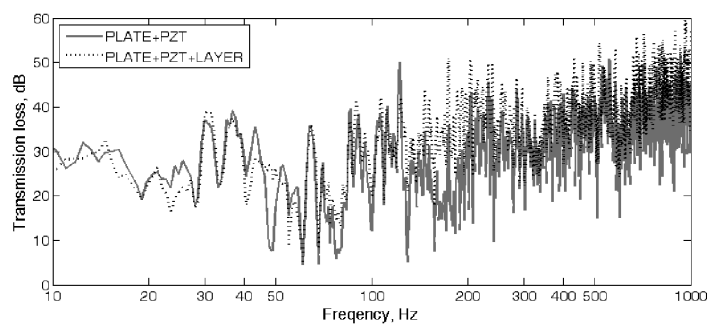

Fig. 4. Transmission loss of both test plates.

are divided into two sets, in which three elements are used as damping actuators and one as a sensor. One of two patches configuration is used in experiment, set $\mathrm{I}$ is near the edges (PZT no. 1, 2, 3, 4) and set II is near the center of the test plate (PZT no. $5,6,7,8$ ) as illustrated in Fig. 5.

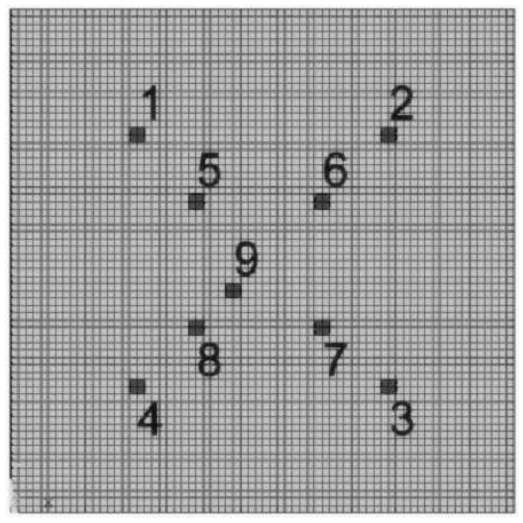

Fig. 5. PZT location on the structure, FE model.

This part of experiment also shows additional layer influence on active structural acoustic control. Active structural acoustic control refers to the process of reducing sound power transmission or radiation from a structure using actuators integrated with the structure itself. In this experiment, PZT tiles serve as actuators to change the vibration distribution of the structure such that the overall sound radiation is reduced. Selection of frequencies to change the vibration distributions is done after numerical computations and FEM simulations of plate resonant frequencies. Acoustic signal at one particular frequency is generated in the sending chamber by a loudspeaker. Then the PZT tiles are actuated to vibrate by applying voltage at specific amplitude and phase.

Experiment was conducted for several particular frequencies but the idea and characteristic of the phenomenon is presented for two frequencies. The first frequency to active structural acoustic control is $54 \mathrm{~Hz}$. This frequency corresponds to 5 th mode shape of square clamped at the edge plate. Figure 2 illustrates the mode shape and location of PZT tiles which exactly match the maximum vibration amplitude on the test plate. This location allowed almost totally to cut off the sound radia- tion at this frequency as shown in Fig. 6 and equalization to the noise level. It can be seen in Figs. 7 to 10 that for plate with elastomer layer the level of sound pressure and vibration is higher than for not layered plate, regardless of whether the active control is turned off or on. Study on elastomer layer application confirms that this kind of passive technique is inefficient at low frequencies and can even worsen active methods effect.

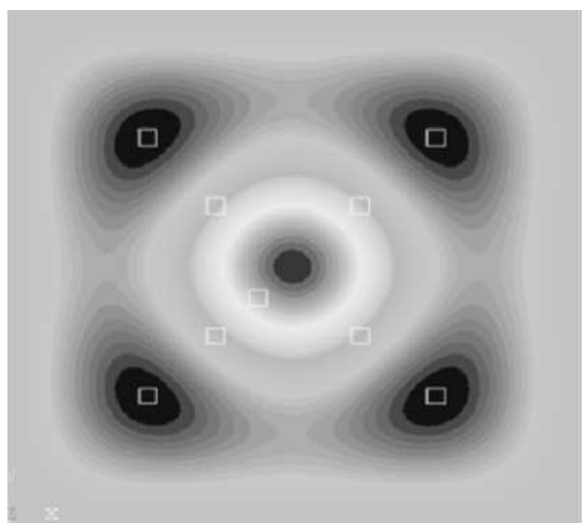

Fig. 6. Mode shape of test plate at $54 \mathrm{~Hz}$.

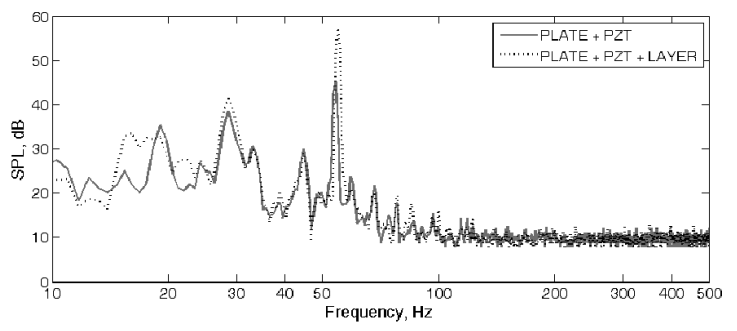

Fig. 7. Sound radiation, active control turned off at $54 \mathrm{~Hz}$.

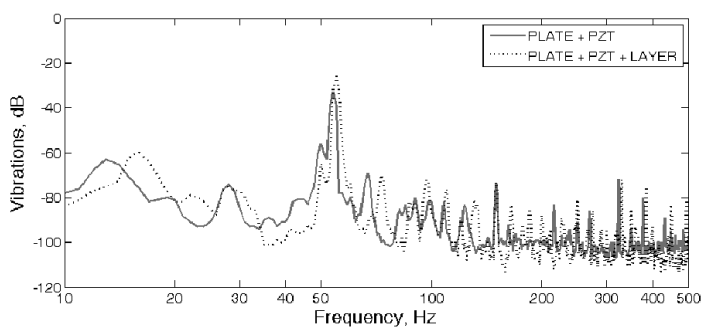

Fig. 8. Vibration, active control turned off at $54 \mathrm{~Hz}$.

Second frequency chosen to operate on was $246 \mathrm{~Hz}$. It is illustrated in Fig. 11 that the maximum amplitude of mode shapes at this frequency does not occur in places where PZT are stuck. Despite the fact, PZT tiles induce vibrating energy which changes mechanical impedance and in consequence reduces acoustic radiation.

Figures 12 to 15 illustrate sound radiation and plate vibration with active control turned on and off. In this case 


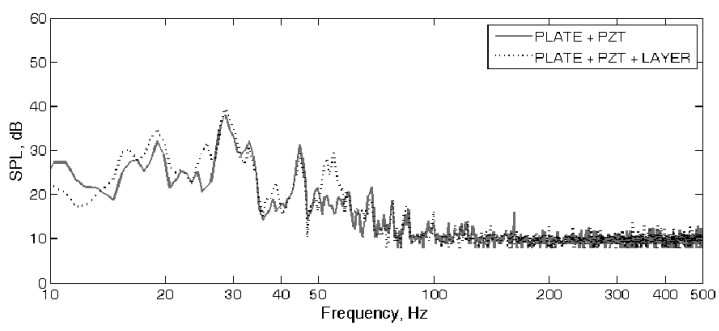

Fig. 9. Sound radiation, active control turned on at $54 \mathrm{~Hz}$.

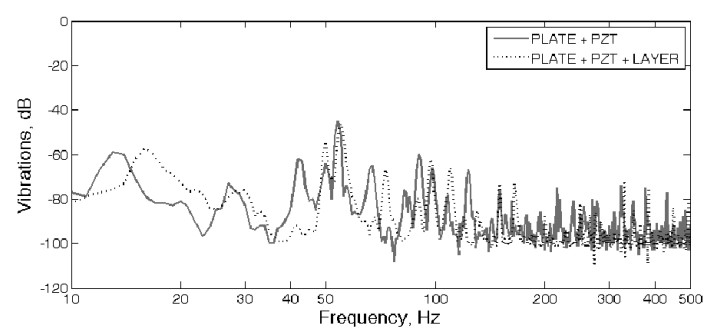

Fig. 10. Vibration, active control turned on at $54 \mathrm{~Hz}$.

the elastomer layer impact on plate radiation is slightly different to the low frequency. At first, vibrations measured for this particular frequency are still higher $(4 \mathrm{~dB})$ for plate with elastomer layer when no active control is applied. At the same time sound radiation has the same level comparing layered and single plate.

In contrary to the first frequency $(54 \mathrm{~Hz})$, when the active control is turned on the sound radiation efficiency decreases more than $3 \mathrm{~dB}$ for the test plate with elastomer layer. Vibration level is also slightly lower $(2 \mathrm{~dB})$ for the situation when PZTs apply bending moment to the test plate and increase its mechanical impedance.

Summing up, all resonant frequencies, in which reduction of sound radiation was achieved are shown in Fig. 16. In most cases, vibration reduction of 7 to $13 \mathrm{~dB}$ gives very satisfactory reduction in sound radiation of 17 to $28 \mathrm{~dB}$.

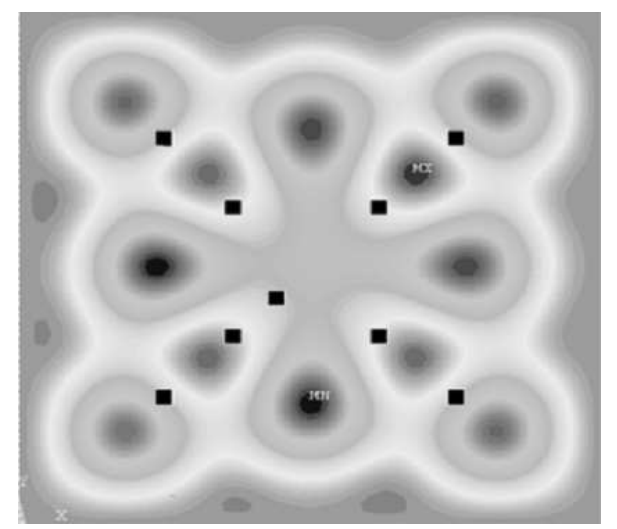

Fig. 11. Mode shape of test plate at $246 \mathrm{~Hz}$.

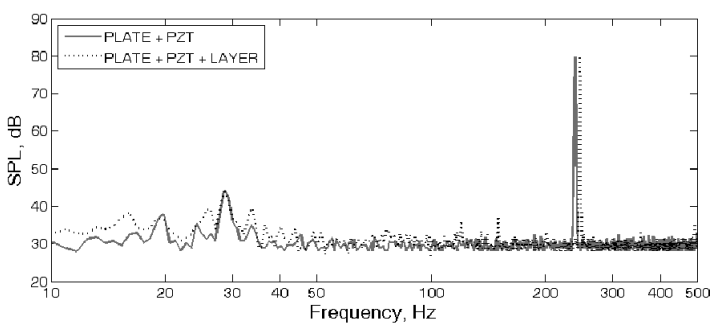

Fig. 12. Sound radiation, active control turned off at $246 \mathrm{~Hz}$.

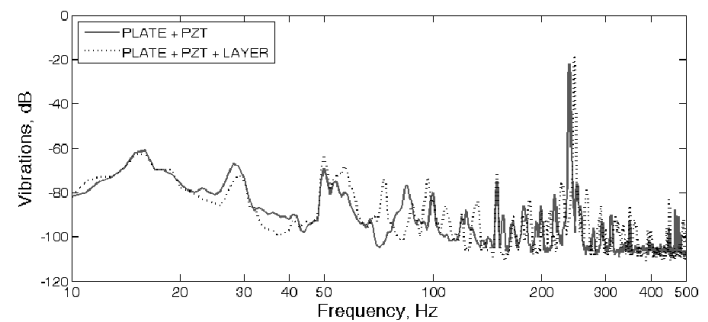

Fig. 13. Vibration, active control turned off at $246 \mathrm{~Hz}$.

In Fig. 17 we can see reduction of vibration and sound radiation for combined structure. Not all resonant frequencies from the steel plate have been examined but comparison for those can be made. Similar situation for the first frequency can be observed, like steel plate; this structure gives better result using external PZTs.

There are also two frequencies when sound radiation reduction is achieved even when the vibration increases. It is assumed that additional mechanical impedance from PZT actuators causes reduction in sound radiation simultaneously increasing vibrations at the same point.

\section{Conclusions}

The feasibility of improving the transmission loss of a lightweight square plate by using PZT patches and elastomer porous layer has been investigated numerically and experimentally. It has been shown that additional layer placed on the plane is too thin and light to increase sound reduction significantly. Modern technology, however, requires a minimum of additional weight in order to improve the acoustic conditions. Actuators like PZT due to

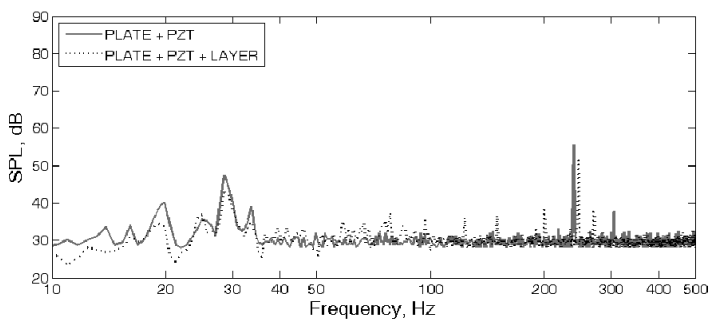

Fig. 14. Sound radiation, active control turned on at $246 \mathrm{~Hz}$. 


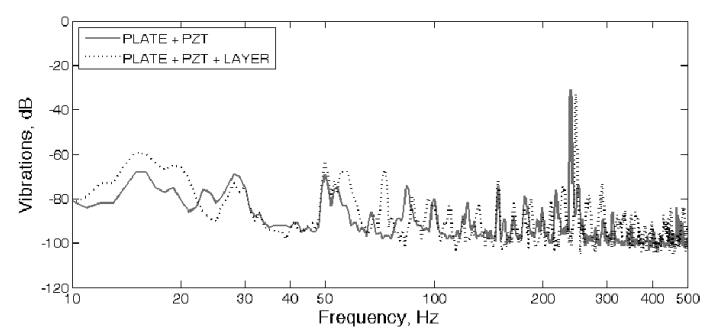

Fig. 15. Vibration, active control turned on at $246 \mathrm{~Hz}$.

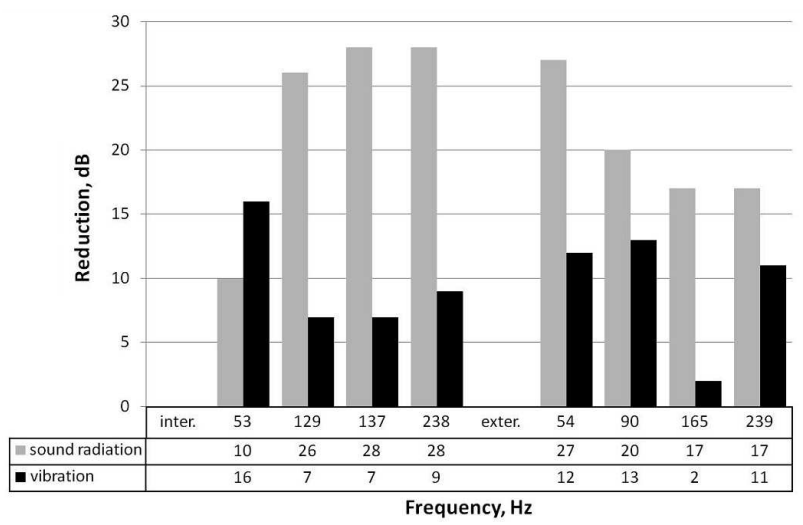

Fig. 16. Steel plate + PZT.

its low mass have marginal impact on structure weight. Combination of both methods can augment some difficulties. As in considered example, the use of one of the PZTs as the actuator element excitation resulted in a problem in exciting some resonant modes. However, the reduction of radiated sound achieved for some particular frequencies was much higher. For example radiation mode about $240 \mathrm{~Hz}$ was minimized much better for plate with elastomer layer; damping via external actuators gave $20 \mathrm{~dB}$ improvement. The resulting frequency characteristics of sound radiation and vibration levels illustrated in the figures show different behaviour for the two configurations

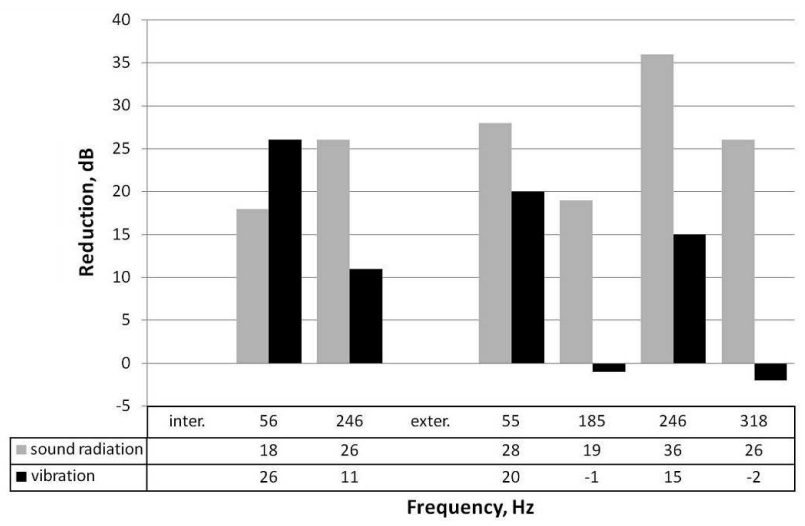

Fig. 17. Steel plate + PZT + elastomer. of test plates.

Generally, at low band frequencies the influence of elastomer layer is negligible or even affects negatively on sound radiation. Transmission loss measurements show some increase in insulation above $400 \mathrm{~Hz}$ but in total it is $1 \mathrm{~dB}(\mathrm{~A})$. The effect on active methods efficiency at resonant frequencies when applying elastomer layer differs slightly and depends probably on frequency shift. To make significant change there should be applied a porous layer of unless 50 to $100 \mathrm{~mm}$ thick. However, a large advantage of this layer is achieved when putting it on the side where PZTs are glued to the test plate. The layer prevents fragile piezoceramic and especially wiring connections from damage. Solution that combines passive and active methods is a structure with porous layer and PZT patches. It is not only the combination of both methods but also application of elastomer layer is a protection coating for PZTs and wiring which are easy to damage. The temperature of vulcanization for elastomer is small enough (less than half of the Curie temperature) to keep the piezoelectric effect in PZT patches. The next stages of research will be based on acoustic excitation and will be more similar to natural conditions.

\section{Acknowledgments}

This research work is supported by the Polish Ministry of Science and Higher Education grant no. N N504 282737.

\section{References}

[1] C.A. Gentry, C. Guigou, C.R. Fuller, J. Acoust. Soc. Am. 101, 1771 (1997).

[2] M.D. Rao, J. Sound Vib. 262, 457 (2003).

[3] C.R. Fuller, S.J. Elliott, P.A. Nelson, Active Control of Vibration, Academic Press, London 1996.

[4] S.J. Elliott, M.E. Johnson, J. Acoust. Soc. Am. 51, 946 (1972).

[5] C.E. Wallace, J. Acoust. Soc. Am. 94, 2194 (1993).

[6] A. Brański, S. Szela, Arch. Control Sci. 17, (2007).

[7] A. Brański, S. Szela, Arch. Acoust. 33, 413 (2008).

[8] M. Kozień, J. Wiciak, Acta Phys. Pol. A 116, 348 (2009).

[9] M. Kozień, J. Wiciak, Arch. Acoust. 33, 643 (2008).

[10] M. Kozupa, Arch. Acoust. 34, 376 (2009).

[11] R. Trojanowski, J. Wiciak, Arch. Acoust. 34, 381 (2009).

[12] R. Filipek, J. Wiciak, Acta Acoust. united with Acoustica 92, 118 (2006). 